\title{
Treatment of Lateral Epicondylitis with Collagen Injections: a Pilot Study
}

\author{
B. Corrado, G. Mazzuoccolo, L. Liguori, V. A. Chirico, M. Costanzo, I. Bonini, \\ G. Bove, L. Curci \\ Department of Public Health, University Federico II of Naples, Italy
}

\section{CORRESPONDING AUTHOR:}

\section{Bruno Corrado}

Physical Medicine and Rehabilitation

Department of Public Health

University Federico II of Naples

Via S. Pansini n.5, 80131

Naples, Italy

Phone: +390817462796

E-mail: bruno.corrado@unina.it

DOI:

10.32098/mltj.04.2019.14

LEVEL OF EVIDENCE: 4

\begin{abstract}
SUMMARY
Background. Lateral epicondylitis, or tennis elbow, is the prevalent cause of elbow pain among adults. Collagen injections are used to treat different musculoskeletal disorders. Type I porcine collagen has proved to enhance tendon repair in vitro. Aims of the present pilot study were to verify the effects of type I porcine collagen injections on pain and disability in patients with tennis elbow and therefore to check if there are grounds for carry out a randomized controlled trial.

Methods. Fifty patients, who have been suffering lateral epicondylitis for at least 6 months, were treated with a series of 5 type I porcine collagen injections, at weekly intervals. The Patient-Rated Tennis Elbow Evaluation questionnaire was employed to verify the effects of collagen injections at 1-month and 3-month follow-up.

Results. As regards the baseline, the total score showed an average reduction of $57 \%$ (55\% in terms of pain and $58 \%$ in terms of function) at 1-month follow-up and an average reduction of $66.1 \%(68.9 \%$ in terms of pain and $63.2 \%$ in terms of function) at 3 -month follow-up. The results were statistically significant $(\mathrm{p}<0.05)$ according to the Kruskal-Wallis test.

Conclusions. Up until now there were no studies about treatment of tennis elbow with collagen injections. Compared to other regenerative injection therapies, collagen injections seemed to be one of the most effective and fast-acting. The positive findings of this pilot study can be the bases for conducting clinical trials with higher level of evidence.
\end{abstract}

\section{KEY WORDS}

collagen; injections; lateral epicondylitis; tendinopatby; tennis elbow

\section{BACKGROUND}

Lateral epicondylitis (LE), also known as tennis elbow, is a frequent painful syndrome of the elbow, due to tendinopathy of the common extensor tendon at the lateral epicondyle of the humerus (1). The prevalence of LE in adults is between $1 \%$ and $3 \%$; it occurs most frequently in the fourth and fifth decades of life, with no gender-related predisposition. As regards etiopathogenesis, causes are not always clearly identified (2). However, the main risk factors are well known: old age, high Body Mass Index (BMI), high total cholesterol levels, previous rotator cuff disease, De Quervain's disease, carpal tunnel syndrome, smoking, and low social status (3). LE is often associated with activities involving repetitive movements, such as grabbing objects, wrist extension, forearm supination or pronation. The most involved muscles in the pathogenesis of $\mathrm{LE}$ are the extensor carpi radialis brevis (ECRB), then the supinator and other extensor muscles such as the extensor carpi radialis longus (ECRL), extensor digitorum (ED), extensor digiti minimi $(\mathrm{EDM})$ and extensor carpi ulnaris (ECU). Despite the name with which this pathology is known, only $5-10 \%$ of patients with lateral epicondylitis actually play tennis (4).

Considering the absence of any inflammatory process in the LE histology findings, the inflammation theory of the disease has been fully rejected (5). At this stage, the majority of the authors consider LE as a degenerative process triggered by a single trauma or several repetitive microtrauma (6). Nirschl first described the LE pathogenesis as angiofibroblas- 
tic proliferation, in which tendons exhibit hyperactivity of fibroblasts, vascular hyperplasia and unstructured collagen fibers (with loss of the physiological compactness and orientation) (7). More recently, it has been shown that LE tendinosis is characterized by the variability in tendon cell density, the extracellular matrix alteration, the presence of chondroid-like proteins, and the increase of water in the tendon structure. Moreover, an increase in matrix metalloproteinases has been described together with anarchic neovascularization and sprouting of small nerve fibers with receptors for substance $P$ in areas that are physiologically almost entirely deprived of them (8). Another histological feature of LE is the reduction of type I collagen within the tendon structure. Type I collagen is gradually replaced by type III collagen, which shows reduced fiber cross-linking and consequent change of the structure of collagen fibrils (chaotic and not linear anymore). Therefore, the tendon will have less resistance to stresses and will be more susceptible to injuries (9). The above-mentioned histological findings are progressive and lead slowly to clinical symptoms, which are, by the way, quite heterogeneous. Mostly patients refer a pain anteriorly or above the lateral epicondyle of the elbow. The pain typically radiates towards the bellies of forearm extensor muscles. Pain may be intermittent, persistent but mild, or severe with functional disability. Often the pain's trigger is the contraction of carpal and digital extensor muscles.

There are several clinical tests for the diagnosis of LE: Maudley's test, Thomson's manoeuvre, the chair lift test and the hand-grip strength evaluation. Imaging can be of use for the diagnosis of LE, above all ultrasounds and Magnetic Resonance (10). The electrodiagnostic evaluation of the posterior interosseous nerve and the elbow $\mathrm{X}$-ray can play an important role in the differential diagnosis (5).

Despite the high prevalence of LE, there is still no agreement about treatment. Following limitation of physical activities and analgesic drugs use, a spontaneous remission of the disease may occur. In the event of symptoms persistence, several treatments are available, both conservative and surgical. The aim of the surgical treatment is the debridement of the angiofibrotic tissue produced during the tendon degenerative process and, eventually, the injured tendons reconstruction. LE conservative treatment includes: rest, physical therapy, braces, medicated plaster (11), Non-Steroidal Anti-Inflammatory Drugs (NSAIDs), Extracorporeal Shock-Wave Therapy (ESWT), percutaneous radiofrequency lesioning, laser therapy, acupuncture, and injection therapy (12). Several injection therapy options are available and described in the medical literature, including injection of: corticosteroids, hyaluronic acid (HA), botulinum toxin, platelet-rich plasma (PRP), tenocyte-like cells derived from cutaneous fibroblasts, and stem cells derived from autolo- gous adipose tissue (12). These injection treatments have shown variable effectiveness and have some criticalities. For example, corticosteroids are useful in the short term, but useless in the long term; PRP or autologous stem cells are expensive and not easy to use.

Collagen injections are used to treat different musculoskeletal disorders (13-15). However, to date no studies on the effectiveness of collagen injections in treating tennis elbow have been published. Biological rationale for the use of type I porcine collagen injections in the treatment of LE was based on the results of the 2018 study by Randelli et al. (16), who stated that type I porcine collagen could induce in tenocytes an anabolic phenotype by stimulating tenocyte proliferation and migration and type I collagen synthesis, maturation, and secretion, thus favoring tendon repair.

The aims of this pilot study were (a) to evaluate the effects of a series of 5 collagen injections (once a week) on pain and disability in a group of patients who have been affected by LE for at least 6 months, and consequently, (b) to investigate whether crucial components of a randomized controlled trial (RCT) will be feasible.

\section{MATERIALS AND METHODS}

This is a prospective observational pilot study and was carried out at Federico II University Hospital in Naples, Italy, Department of Rehabilitation and Orthopedics. The subjects were all outpatients and we enrolled them from January 2017 to October 2018. All the patients who referred symptoms of LE was evaluated to verify the criteria for the enrollment. The inclusion criteria were: (a) age $>18$ years, (b) clinical symptoms of LE for at least 6 months, (c) lack of therapy in the last 6 months, (d) pain triggered by pression on lateral epicondyle (on proximal insertion of the common extensor tendon), (e) positive Maudley's test and Thomson's manoeuvre, (f) absence of bone lesions on plain X-ray. The exclusion criteria are listed in table I. After a full and clear description of the study protocol, all patients enrolled were invited to sign the informed consent. The study was carried out in accordance with the principles of the Declaration of Helsinki and meets the ethical standards of the journal (17) and of the local ethics committee as well.

We enrolled 50 patients, of which 33 males and 17 females, with an average age of $52.25 \pm 13.25$ years. For the treatment we planned five injections of $2 \mathrm{ml}$ porcine type I collagen, once a week. Injections were performed using the palpatory technique at the level of the proximal insertion of the wrist and fingers extensor tendons on the lateral epicondyle. The palpatory technique has proved to be accurate (18) and with the same effectiveness of the echo-guided method (19). No other treatment has been associated with collagen injections. 
Table I. Exclusion criteria.

\begin{tabular}{l}
\hline Traumatic elbow injuries in the previous $\mathbf{6}$ weeks \\
\hline Elbow instability \\
\hline Previous surgery of the elbow \\
\hline Any other pathology affecting the same arm \\
\hline Cervicobrachial pain syndrome \\
\hline Contraindications to injection therapy \\
\hline Any other therapy for epicondylitis in the last 6 months \\
\hline Hemorrhagic diathesis or anticoagulant therapy \\
\hline Local or systemic infections \\
\hline Diabetes or autoimmune diseases \\
\hline Obesity (Body Mass Index $\geq 30)$ \\
\hline Definite chronic hyperglicemia $(\mathrm{HbA} 1 \mathrm{c} \geq 6.5 \%)$ \\
\hline Hypercholesterolemia (total cholesterol levels $\geq 240 \mathrm{mg} / \mathrm{dL}$ ) \\
\hline Definite hypertension (SBP $\geq 140 \mathrm{mmHg}$ or $\mathrm{DBP} \geq 85 \mathrm{mmHg})$ \\
\hline Pregnancy or feeding time \\
\hline Psychiatric disorders
\end{tabular}

Patients were evaluated at the time of enrollment (T0), and one month (T1) and three months (T2) after the last injection by means of the Patient-Rated Tennis Elbow Evaluation (PRTEE) questionnaire. The PRTEE was introduced in clinical practice by MacDermid in 2005 (20). It is a self-compiled 15-item questionnaire that assesses pain and disability in patients with tennis elbow. The PRTEE consists of 2 subscales: pain (5 items) and function (10 items). The function subscale, in its turn, includes usual activities (4 items) and specific activities (6 items). Each item has a score between 0 (no pain or disability) and 10 (the worst possible pain or complete disability). The total score goes from 0 to 100 , with the highest scores indicating the worse situation in terms of pain and disability. Pain and function are equally represented in the score. The PRTEE questionnaire is highly reliable, reproducible and sensitive (21). In the present study we used the 2012 Italian version of the PRTEE questionnaire (22).

\section{RESULTS}

The Kruskal-Wallis test and the Dunn's post-hoc analysis were employed for the statistical analysis. The confidence interval was established at $95 \%(\mathrm{p}<0.05)$. Both the average total score on the PRTEE questionnaire and the average scores of the pain and function subscales have been taken into consideration. At the time of enrollment (T0) the average scores of the PRTEE questionnaire were: (a) total score $68.42 \pm 16.50$, (b) pain score $35.6 \pm 7.57$, and (c) function score $32.82 \pm 9.96$. At T1 follow-up (1 month after last injection) the scores were: (a) total score $29.74 \pm 16.95$, (b) pain score $15.9 \pm 8.9$, function score $13.84 \pm 9.33$. At T2 follow-up ( 3 months after last injection) the scores were: (a) total score $23.17 \pm 13.68$, (b) pain score $11.08 \pm 6.08$, function score $12.09 \pm 8.9$. The Kruskal-Wallis test provided very strong evidence $(p=0.000)$ for all the three variables analysed (pain score, function score and total score). The Dunn's post-hoc tests were carried out in order to analyse the differences between the three pairs of groups (T0-T1, T0-T2, and T1-T2) for each variable. There was always very strong evidence $(p=0.000)$ of a difference between the group T0 and the group T1 and between the group T0 and the group T2. By contrast, there was never evidence of a difference between the group T1 and the group T2 (p $>0.05$ ). As regards the score's variations between T0 and $\mathrm{T} 1$, we observed a $55 \%$ reduction in the pain score, a $58 \%$ reduction in the function score, and a $57 \%$ reduction in the total score. Finally, with reference to the score's variations between $\mathrm{T} 0$ and $\mathrm{T} 2$, a $68.9 \%$ reduction in the pain score, a $63.2 \%$ reduction in the function score and a $66.1 \%$ reduction in the total score were registered. No adverse event has been described after collagen injections, except for some cases of burning sensation at the injection site which resolved spontaneously in a few hours.

All the results are summarized in figures 1, 2 and 3.

\section{DISCUSSION}

LE is the most frequent cause of elbow pain in adults and, from the etiological point of view, it can be defined as an angiofibroblastic tendinosis. Currently there isn't a consensus regarding the treatment of LE. Many conservative therapeutic approaches have been proposed, both systemic and local, but until now, few of them have valid clinical evidence. Injection therapy is one of the most studied therapeutic approach. This treatment allows the drug to reach the tendon directly, increasing drug effectiveness and reducing systemic side effects. In the recent literature some drugs have proved to be particularly effective in treating LE if administered by infiltration: HA, PRP, dextrose (prolotherapy), high volume $0,9 \%$ saline solution and botulinum toxin.

At this stage there aren't in literature studies about collagen injection in the treatment of LE. Therefore, in this pilot study we wanted to evaluate the collagen injection therapy in a cohort of 50 subjects affected by tennis elbow ( 5 injections, once a week, of type I porcine collagen). The results were evaluated by administering the PRTEE questionnaire, before the first injection, and one month and three months after the last injection. We observed a $57 \%$ reduction in the PRTEE total score at T1 follow-up $55 \%$ in the PRTEE 


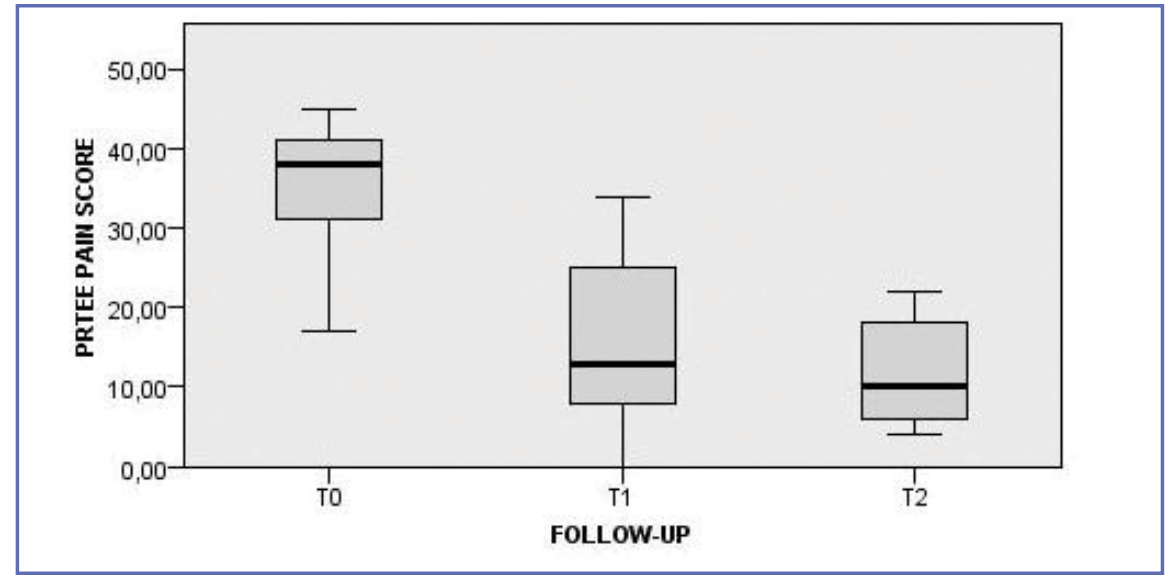

Figure 1. PRTEE function subscale score at baseline (TO), and 1 month (T1) and 3 months (T2) after the last injection.

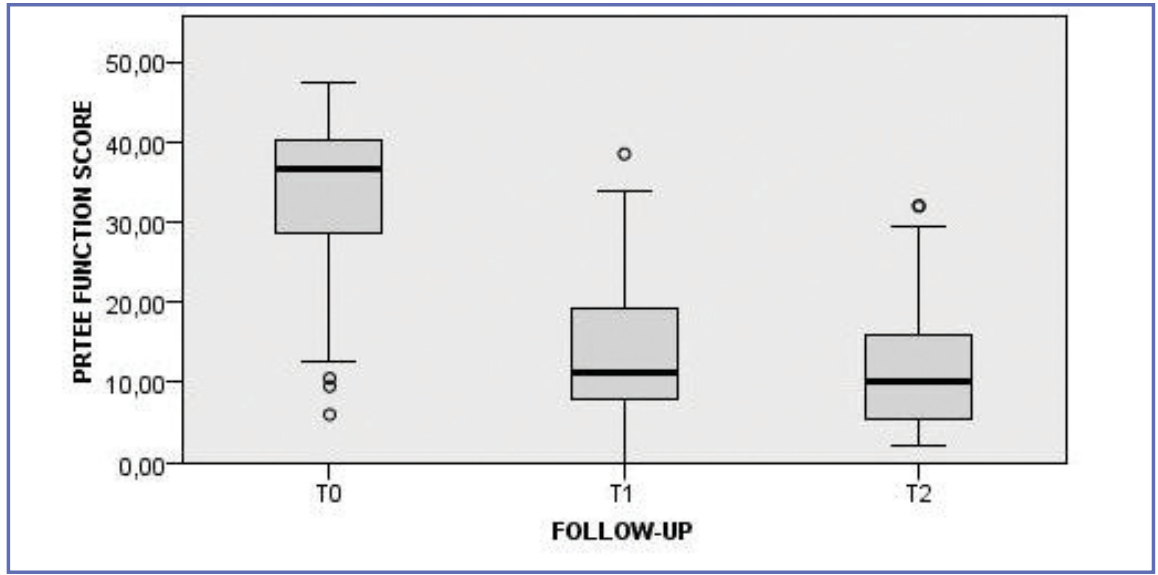

Figure 2. PRTEE pain subscale score at baseline (TO), and 1 month (T1) and 3 months (T2) after the last injection.

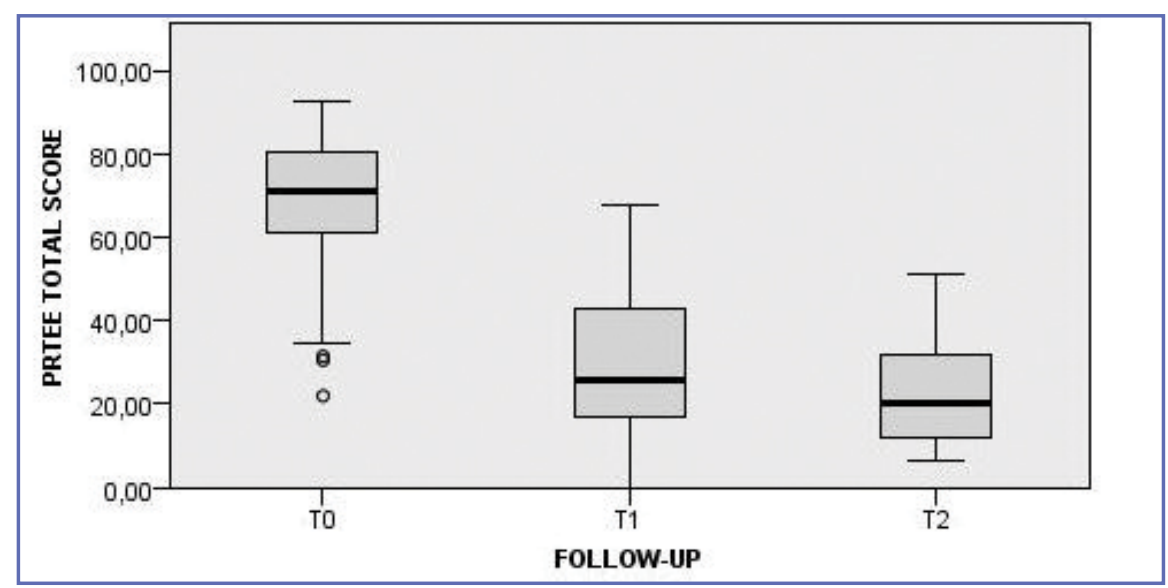

Figure 3. PRTEE total score at baseline (TO), and 1 month (T1) and 3 months (T2) after the last injection. pain score and $58 \%$ in the PRTEE function score, respectively). Finally, three months after the last injection, the PRTEE total score achieved a $66.1 \%$ reduction $(68.9 \%$ as regards the pain score and $63.2 \%$ as regards the function score).

Since there are no studies on this topic in the literature, we cannot make a direct comparison.

However, it seemed appropriate to compare our results with other studies in which the effectiveness of regenerative injection therapies has been evaluated, such as the injection of hypertonic dextrose and sodium morruate (prolotherapy), the injection of HA and chondroitin sulfate (CS), and finally the injection of PRP associated with a scaffold of human collagen. All the considered studies used the PRTEE questionnaire for the evaluation of results. Therefore, we could make the comparison.

Rabago et al. in 2013 carried out a randomized controlled trial in order to test the effectiveness of injection of hypertonic dextrose and sodium morruate (prolotherapy) in the treatment of tennis elbow (23). Nine patients were treated with prolotherapy and the results were compared with those obtained in a homogeneous control group treated with the "waitand-see" approach. The average total score of the PRTEE questionnaire in the treated group was reduced by $5.2 \%$ after 4 weeks, by $23.8 \%$ after 6 weeks, by $53.5 \%$ after 16 weeks and by $74.9 \%$ after 32 weeks. The differences with the results obtained in the control group were statistically significant at 6 and at 12 weeks, while no statistically significant differences were found at 4 weeks. The control group did not carry out the follow-up evaluation at 32 weeks.

In their 2015 prospective randomized controlled trial, Tosun et al. evaluated the effects of a single $\mathrm{HA}+\mathrm{CS}$ injection in 25 patients with LE and compared the results with a single cortisone + 
anesthetic injection (control group)(24). In the treated group the authors reported a $51.35 \%$ of mean total score reduction after 3 months and a $61.72 \%$ after 6 months. The difference between the two groups was statistically significant only at the third month follow-up evaluation.

In a recent paper (2019), Farkash et al. showed good results in LE treatment with a single PRP injection plus a scaffold of human collagen (25). Forty patients were enrolled and the results showed a $34 \%$ reduction in the average score at 1 month and a $59 \%$ at 6 months, compared to baseline values. We can compare our results at 1-month follow-up with those achieved in the studies by Rabago and Farkash. In the aforementioned trials we can see an average total score reduction of $5.2 \%$ and $34 \%$, respectively, while in our cohort study we obtained a reduction of $57 \%$. In his study, Tosun described a $51.35 \%$ reduction after a 3 -month follow up. This result is lower than the one obtained in our sample after the same time of follow-up (66.1\%). We underline that the 5 collagen injections, proposed in our therapeutic approach, have obtained better results at 1 month than the single injection of dextrose + morruate and PRP + collagen scaffold (higher difference in the first and less in the second case) and at the same time our protocol

\section{REFERENCES}

1. Longo UG, Franceschetti E, Rizzello G, Petrillo S, Denaro V. Elbow tendinopathy. Muscles Ligaments Tendons J. 2012;2:115-120.

2. Shiri R, Viikari-Juntura E, Varonen H, Heliövaara M. Prevalence and determinants of lateral and medial epicondylitis: a population study. Am J Epidemiol. 2006;164:1065-1074.

3. Lee SH, Gong HS, Kim S, Kim J, Baek GH. Is there a relation between lateral epicondylitis and total cholesterol levels? Arthroscopy. 2019;35:1379-1384. doi: 10.1016/j. arthro.2019.01.048.

4. Cohen M, da Rocha Motta Filho G. Lateral epicondylitis of the elbow. Rev Bras Ortop. 2012;47:414-420. doi: 10.1016/S22554971(15)30121-X.

5. Vaquero-Picado A, Barco R, Antuña SA. Lateral epicondylitis of the elbow. EFORT Open Rev. 2016;1:391-397. doi: 10.1302/2058-5241.1.000049.

6. De Smedt T, de Jong A, Leemput WV, et al. Lateral epicondylitis in tennis: update on aetiology, biomechanics, and treatment. Br J Sports Med. 2007;41:816-819.

7. Richer N, Marchand AA, Descarreaux M. Management of chronic lateral epicondylitis with manual therapy and local cryostimulation: A Pilot Study. J Chiropr Med. 2017;16:279288. doi: 10.1016/j.jcm.2017.07.001.

8. Xu Y, Murrell GAC. The basic science of tendinopathy. Clin Orthop Relat Res. 2008;466:1528-1538. doi: 10.1007/s11999008-0286-4.

9. Riley GP, Harrall RL, Constant CR, Chard MD, Cawston TE, Hazleman BL. Tendon degeneration and chronic shoulder showed greater effectiveness than a single HA + CS injection after a 3 -month follow-up.

The present study has several limitations: (a) a relatively small sample, (b) the lack of a control group, (c) the LE diagnosis based solely on history, physical examination and X-ray, and (d) the use of a subjective evaluation tool. However, it should be emphasized that this is a pilot study, and its objective was to evaluate the feasibility of a subsequent randomized controlled clinical trial. To date indeed, this is the first study in the literature on the effectiveness of collagen injection therapy in tennis elbow.

In conclusion, this pilot study has shown that a series of 5 collagen injections, at weekly intervals, is able to reduce significantly pain symptoms and improve the function in a very short time (1 month), in a group of 50 patients with LE. Moreover, we stated that the good results further increase two months later. Therefore, we can conclude that there are grounds for carrying out a RCT to confirm our preliminary data.

\section{CONFLICT OF INTERESTS}

The authors declare that they have no conflicts of interest. pain: changes in the collagen composition of the human rotator cuff tendons in rotator cuff tendinitis. Ann Rheum Dis. 1994;53:359-366. doi: 10.1136/ard.53.6.359.

10. Cha YK, Kim SJ, Park NH, Kim JY, Kim JH, Park JY. Magnetic resonance imaging of patients with lateral epicondylitis: Relationship between pain and severity of imaging features in elbow joints. Acta Orthop Traumatol Turc. 2019;S1017995X:30499-30501. doi: 10.1016/j.aott.2019.04.006. [Epub ahead of print].

11. Frizziero A, Causero A, Bernasconi S, et al. Efficacy of betamethasone valerate medicated plaster on painful chronic elbow tendinopathy: a double-blind, randomized, placebo-controlled trial. Muscles Ligaments Tendons J. 2016;6(1):131-139. doi: 10.11138/mltj/2016.6.1.131.

12. Lai WC, Erickson BJ, Mlynarek RA, Wang D. Chronic lateral epicondylitis: challenges and solutions. Open Access J Sports Med. 2018;9:243-251. doi: 10.2147/OAJSM.S160974.

13. Furuzawa-Carballeda J, Lima G, Llorente L, et al. Polymerized-type I collagen downregulates inflammation and improves clinical outcomes in patients with symptomatic knee osteoarthritis following arthroscopic lavage: a randomized, double-blind, and placebo-controlled clinical trial. ScientificWorldJournal. 2012;2012:342854. doi: $10.1100 / 2012 / 342854$.

14. Martin Martin LS, Massafra U, Bizzi E, Migliore A. A double blind randomized active-controlled clinical trial on the intra-articular use of Md-Knee versus sodium hyaluronate in patients 
with knee osteoarthritis (“Joint”). BMC Musculoskelet Disord. 2016;17:94. doi: 10.1186/s12891-016-0948-4.

15. Nitecka-Buchta A, Walczynska-Dragon K, Batko-Kapustecka J, Wieckiewicz M. Comparison between collagen and lidocaine intramuscular injections in terms of their efficiency in decreasing myofascial pain within masseter muscles: a randomized, single-blind controlled trial. Pain Res Manag. 2018;2018:8261090. doi: 10.1155/2018/8261090.

16. Randelli F, Menon A, Giai Via A, et al. Effect of a collagen-based compound on morpho-functional properties of cultured human tenocytes. Cells. 2018;7(12). pii: E246. doi: 10.3390/cells7120246.

17. Padulo J, Oliva F, Frizziero A, Maffulli N. Muscles, Ligaments and Tendons Journal - Basic principles and recommendations in clinical and field Science Research: 2018 update. MLTJ 2018;8:305-307. doi: 10.32098/mltj.03.2018.01.

18. Koski JM, Hermunen HS, Kilponen VM, Saarakkala SJ, Hakulinen UK, Heikkinen JO. Verification of palpation-guided intra-articular injections using glucocorticoid-air-saline mixture and ultrasound imaging (GAS-graphy). Clin Exp Rheumatol. 2006;24:247-252.

19. Malahias MA, Kaseta MK, Kazas ST, Megaloikonomos PD, Mavrogenis AF, Babis GC. Image-guided versus palpation-guided injections for the treatment of chronic lateral epicondylopathy: a randomized controlled clinical trial. Handchir Mikrochir Plast Chir. 2018;50:348-352. doi: 10.1055/a-0732-5556.

20. Macdermid J. Update: the Patient-rated Forearm Evaluation Questionnaire is now the Patient-rated Tennis Elbow Evaluation. J Hand Ther. 2005;18:407-410.
21. Rompe JD, Overend TJ, MacDermid JC. Validation of the Patient-rated Tennis Elbow

22. Evaluation Questionnaire. J Hand Ther. 2007;20:3-10. doi: 10.1197/j.jht.2006.10.003.

23. Cacchio A, Necozione S, MacDermid JC, et al. Cross-cultural adaptation and measurement properties of the italian version of the Patient-Rated Tennis Elbow Evaluation (PRTEE) questionnaire. Phys Ther. 2012;92:1036-1045. doi: 10.2522/ ptj.20110398.

24. Rabago D, Lee KS, Ryan M, et al. Hypertonic dextrose and morrhuate sodium injections (prolotherapy) for lateral epicondylosis (tennis elbow): results of a single-blind, pilot-level, randomized controlled trial. Am J Phys Med Rehabil. 2013;92:587-596. doi: 10.1097/PHM.0b013e31827d695f.

25. Tosun HB, Gumustas S, Agir I, et al. Comparison of the effects of sodium hyaluronate-chondroitin sulphate and corticosteroid in the treatment of lateral epicondylitis: a prospective randomized trial. J Orthop Sci. 2015;20:837-843. doi: 10.1007/s00776-015-0747-z.

26. Farkash U, Avisar E, Volk I, et al. First clinical experience with a new injectable recombinant human collagen scaffold combined with autologous platelet-rich plasma for the treatment of lateral epicondylar tendinopathy (tennis elbow). J Shoulder Elbow Surg. 2019;28:503-509. doi: 10.1016/j. jse.2018.09.007. 